\title{
INDEX OF RIGIDITY OF DIFFERENTIAL EQUATIONS AND EULER CHARACTERISTIC OF THEIR SPECTRAL CURVES
}

\author{
KAZUKI HIROE \\ CHIBA UNIVERSITY
}

\begin{abstract}
We show a coincidence of index of rigidity of differential equations with irregular singularities on a compact Riemann surface and Euler characteristic of the associated spectral curves which are recently called irregular spectral curves. Also we present a comparison of local invariants, so called Milnor formula which links the Komatsu-Malgrange irregularity of differential equations and Milnor number of the spectral curves.
\end{abstract}

\section{INTRODUCTION}

Higgs bundles with irregular singularities have been studied by many researchers from several points of view, mirror symmetry, geometric Langlands program, nonablelian Hodge correspondence and so on, see [4, 22], 26], [32], 35]. At the same time, studies of relations between spectral curves and differential equations recently increase in importance, see [9], [10] in which differential equations obtained as a quantization of spectral curves are discussed.

This paper presents a numerical comparison between cohomology groups of a differential equation with irregular singularities on a Riemann surface and those of associated spectral curve. A main result in this paper is the following. We consider a differential equation

$$
d w=A w
$$

where $A$ is a square matrix of size $n$ whose entries are meromorphic 1-forms on a compact Riemann surface $X$ of genus $g$. In particular this differential equation is allowed to have several regular/irregular singular points $a_{1}, a_{1}, \ldots, a_{k}$ on $X$. This differential equation defines a lambda connection $\nabla_{\lambda}$ on the trivial bundle $\mathcal{O}_{X}^{\oplus n}$ by

$$
\nabla_{\lambda}=\lambda d-A
$$

for $\lambda \in \mathbb{C}$, and we obtain a (possibly irregular) singular Higgs bundle $\nabla_{0}=A$ as a classical limit of the differential equation. We can define a divisor on

2020 Mathematics Subject Classification. 14H70, 14H20, 34M35.

Key words and phrases. Irregular singularity, Spectral curve, Index of rigidity, Euler characteristic Komatsu-Malgrange irregularity, Milnor number.

The author is supported by JSPS GGrant-in-Aid for Scientific Research (C) Grant Number 20K03648. 
the cotangent bundle $T^{*} X$ as the zero locus of the characteristic polynomial of the Higgs bundle $\nabla_{0}$,

$$
\operatorname{det}\left(y I_{n}-A\right),
$$

and this is called spectral curve $C_{A}$. Singular points appear as poles of $A$ and the zero locus of the characteristic polynomial will pass through the line at infinity $y=\infty$. Thus it is natural to consider the spectral curve as a divisor on a compactified cotangent bundle $\overline{T^{*} X}$. Let us assume one of the following conditions is satisfied at each singular points $a_{1}, a_{2}, \ldots, a_{k}$.

(1) The Hukuhara-Turrittin-Levelt normal form of the germ $A_{a_{i}}$ of $A$ at $a_{i}$ is multiplicity free (see Definition 3.2).

(2) The germ $A_{a_{i}}$ is regular semisimple over $\mathbb{C} \llbracket z_{a_{i}} \rrbracket$ (see Definition 3.9 $)$. Then we can show the following coincidence of the index of rigidity of the differential equation and the Euler characteristic of the spectral curve.

Theorem 0.1 (Theorem 4.5, Corollary 4.6). Let $\nabla_{A}$ be the algebraic connection defined by the differential equation $d w=A w$. Suppose that $C_{A}$ is irreducible. Moreover suppose that $C_{A}$ is smooth on $T^{*} X$. Then the index of rigidity $\operatorname{rig}\left(\nabla_{A}\right)$ of $\nabla_{A}$ and the Euler characteristic $\chi\left(\widetilde{C_{A}}\right)$ of the normalization $\widetilde{C_{A}}$ of $C_{A}$ coincide with each other, i.e.,

$$
\operatorname{rig}\left(\nabla_{A}\right)=\chi\left(\widetilde{C_{A}}\right) .
$$

Moreover assume that $\nabla_{A}$ is irreducible. Then we have the numerical coincidences of cohomology groups,

$$
h_{\mathrm{dR}}^{i}\left(X, j_{! *}\left(\mathcal{E} \operatorname{nd} \nabla_{A}\right)\right)=h^{i}\left(\widetilde{C_{A}}, \mathbb{C}\right), \quad i=0,1,2 .
$$

Here $h^{i}(*):=\operatorname{dim}_{\mathbb{C}} H^{i}(*)$.

This fact has been known by Kamimoto [16] and Oshima 28] for Fuchsian differential equations on $\mathbb{P}^{1}$.

Let us look at the equation

$$
h_{\mathrm{dR}}^{1}\left(X, j_{! *}\left(\mathcal{E} \operatorname{nd} \nabla_{A}\right)\right)=h^{1}\left(\widetilde{C_{A}}, \mathbb{C}\right)
$$

in our main theorem. This can be seen as an analogy of the well-known fact on the infinitesimal deformations of a holomorphic Higgs bundle: the genus of the corresponding spectral curve is equal to half of the dimension of the space of the infinitesimal deformations, see [13] and [27]. That is to say, cohomology group $H_{\mathrm{dR}}^{1}\left(X, j_{! *}\left(\mathcal{E} \mathrm{nd} \nabla_{A}\right)\right)$ is known to be identified with the space of isotipical infinitesimal deformations of $\nabla_{A}$ by THEOREM 4.10 in [5] and also see Lemma 4.7 in [1. Here the isotipical deformation means the deformation of $\nabla_{A}$ under the condition that the HTL-normal forms at $a_{i}, i=1,2, \ldots, k$ are kept fixed. Thus we may say that the main theorem gives an analogy of the fact for holomorphic Higgs bundles to irregular meromorphic connections following the philosophy of the nonabelian Hodge correspondence [31, [4.

This main theorem is a consequence of the following local study of the singularities of the spectral curve. As it is pointed out in 222] and [32], the spectral curve $C_{A}$ has intersections with the line at infinity $X_{\infty}=\overline{T^{*} X} \backslash T^{*} X$ at $\infty_{a_{i}}=\left(\infty, a_{i}\right), i=1,2, \ldots, k$ and these intersection points may have singularities resulting from the irregular singularities of the corresponding 
differential equation and the Higgs bundle. We investigate the singularities of the irregular spectral curve and show that the Milnor number of $C_{A}$ at $\infty_{a_{i}}$ can be computed from the Komatsu-Malgrange irregularity of the corresponding local differential module $M_{A_{a_{i}}}$ as follows.

Theorem 0.2 (Theorem 4.3). The Milnor number of $C_{A}$ at $\infty_{a_{i}}$ for each $i=1,2, \ldots, k$ is

$$
\mu\left(C_{A}\right)_{\infty_{a_{i}}}=-\delta\left(\operatorname{End}_{\mathbb{C}\left(\left\{z_{a_{i}}\right\}\right)}\left(M_{A_{a_{i}}}\right)\right)-r_{C_{A_{a_{i}}}}+2(n-1)\left(C_{A}, X_{\infty}\right)_{\infty_{a_{i}}}+1 .
$$

Here $\left(C, C^{\prime}\right)_{\infty_{a}}$ is the intersection number of divisors $C, C^{\prime}$ at $\infty_{a}, r_{C_{A_{i}}}$ is the number of branches of the germ $C_{A_{a_{i}}}$ and

$$
\begin{aligned}
\delta\left(\operatorname{End}_{\mathbb{C}(\{z\})}\left(M_{A_{a}}\right)\right)= & \operatorname{rank} \operatorname{End}_{\mathbb{C}\left(\left\{z_{a}\right\}\right)}\left(M_{A_{a}}\right)+\operatorname{Irr}\left(\operatorname{End}_{\mathbb{C}\left(\left\{z_{a}\right\}\right)}\left(M_{A_{a}}\right)\right) \\
& -\operatorname{dim}_{\mathbb{C}} \operatorname{Hom}_{\mathbb{C}\left(\left(z_{a}\right)\right)}\left(\operatorname{End}_{\left.\mathbb{C}\left(\left(z_{a}\right)\right)\right)}\left(\widetilde{M_{A_{a}}}\right), \mathbb{C}\left(\left(z_{a}\right)\right)\right)^{\text {hor }} .
\end{aligned}
$$

By using the $\delta$-invariant of a singularity of a plane curve germ, this formula can be written in a simpler form.

Corollary 0.3 (Remark 4.4).

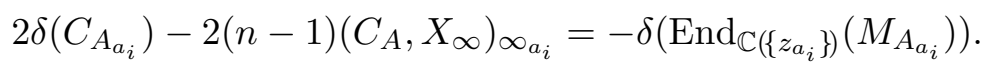

Acknowledgements. The author would like to express his gratitude to Professors Shingo Kamimoto and Toshio Oshima who gave him many inspirations through fruitful discussions. The essential part of the idea to prove the main theorem is based on their pioneering works in the case of Fuchsian differential equations on $\mathbb{P}^{1}$. The most part of this work had been done when the author was a member of the Department of Mathematics in Josai University and the work would have never been completed without the support from Josai University. Finally the author would like to thank Professor Akane Nakamura. Many discussions with her were very much inspiring and encouraging.

\section{Spectral curves of Differential equations}

1.1. Compactified cotangent bundle on a Riemann surface. Let $X$ be a compact Riemann surface of genus $g$ and consider a compactification of $T^{*} X$ defined by

$$
\overline{T^{*} X}:=\mathbb{P}\left(\mathcal{O}_{X} \oplus T^{*} X\right)
$$

which is the projective bundle of the vector bundle $\mathcal{O}_{X} \oplus T^{*} X$. The complement of $T^{*} X$ is denoted by $X_{\infty}:=\overline{T^{*} X} \backslash T^{*} X$. The natural projection

$$
\pi: \overline{T^{*} X} \rightarrow X
$$

enables us to regard this surface as a ruled surface. Thus the Neron-Severi group NS $\overline{T^{*} X}:=\operatorname{Pic} \overline{T^{*} X} / \mathrm{Pic}^{0} \overline{T^{*} X}$ is generated by $X_{0}$, the zero section of $T^{*} X$ and a fiber $f$, namely,

$$
\mathrm{NS} \overline{T^{*} X} \cong \mathbb{Z} X_{0} \oplus \mathbb{Z} f \text {. }
$$

This lattice has the $\mathbb{Z}$-bilinear form determined by the intersection numbers of generators,

$$
\left(X_{0}, f\right)=1, \quad(f, f)=0, \quad\left(X_{0}, X_{0}\right)=-\operatorname{deg} T^{*} X=2 g-2 .
$$




\subsection{Spectral curve of differential equation. Let}

$$
D:=\left\{a_{1}, a_{2}, \ldots, a_{p}\right\} \subset X
$$

be a finite set. We consider a differential equation with poles on $D$,

$$
d w=A w
$$

where $A \in M\left(n, \Omega_{X}(* D)(X)\right)$. We may assume that the set of all poles of $A$ is exactly $D$.

Let us define the spectral curve of this differential equation through the notion of the lambda connection as below. For $\lambda \in \mathbb{C}, \nabla_{\lambda}:=\lambda d-A$ defines a lambda connection on the trivial bundle $\mathcal{O}_{X}^{\oplus n}$, i.e., a $\mathbb{C}$-linear map

$$
\nabla_{\lambda}: \mathcal{O}_{X}^{\oplus n} \rightarrow \mathcal{O}_{X}^{\oplus n} \otimes \Omega_{X}(* D)
$$

satisfying

$$
\nabla_{\lambda}(f v)=\lambda d f \otimes v+f \otimes \nabla_{\lambda} v
$$

for $f \in \mathcal{O}_{X}, v \in \mathcal{O}_{X}^{\oplus n}$. Then the spectral curve $C_{A}$ is a divisor on $\overline{T^{*} X}$ defined as the zero locus of the charachterisitic polynomial of the Higgs bundle $\left(\mathcal{O}_{X}^{\oplus n}, \nabla_{0}\right)$ in the following way. First let us take a complex atlas $\left\{\left(U_{i}, z_{i}\right)\right\}_{i=1,2, \ldots, k}$ of $X$ with an open covering $X=\bigcup_{i=1}^{k} U_{i}$ and local coordinates $z_{i}: U_{i} \rightarrow \mathbb{C}$. On this local coordinate system the canonical 1-form $\theta \in \Omega_{T^{*} X}\left(T^{*} X\right)$ can be expressed as $\theta=\eta_{i} d z_{i}$ with the fiber coordinate $\eta_{i}$ of $T^{*} X$ on $U_{i}$ for $i=1,2, \ldots, k$. Then the canonical 1 -form $\theta$ extends to the meromorphic 1 -form $\bar{\theta}$ on $\overline{T^{*} X}$ of the form

$$
\bar{\theta}=\frac{\eta_{i}}{\zeta_{i}} d z_{i}, \quad i=1,2, \ldots, k,
$$

where $\left[\zeta_{i}: \eta_{i}\right] \in \mathbb{P}^{1}$ is the fiber coordinate of $\overline{T^{*} X}=\mathbb{P}\left(\mathcal{O}_{X} \oplus T^{*} X\right)$ on $U_{i}$.

Let us denote the trivialization of $A$ on $U_{i}$ by $A=A_{i}\left(z_{i}\right) d z_{i}, A_{i}\left(z_{i}\right) \in$ $M\left(n, \mathbb{C}\left(z_{i}\right)\right)$. Then the pullback $\pi^{*} A$ by the projection $\pi: \overline{T^{*} X} \rightarrow X$ can be written in the same form

$$
\pi^{*} A\left(\left[\zeta_{i}: \eta_{i}\right], z_{i}\right)=A_{i}\left(z_{i}\right) d z_{i}
$$

on $\pi^{-1}\left(U_{i}\right) \cong \mathbb{P}^{i} \times U_{i}$.

Then $\operatorname{det}\left(\frac{\eta_{i}}{\zeta_{i}} I_{n}-A_{i}\left(z_{i}\right)\right)$ gives an meromorphic function of $\pi^{-1}\left(U_{i}\right)$ for each $i=1,2, \ldots, k$. Since the compatibility follows immediately from the definition, the collection

$$
\left\{\left(\pi^{-1}\left(U_{i}\right), \operatorname{det}\left(\frac{\eta_{i}}{\zeta_{i}} I_{n}-A_{i}\right)\right)\right\}_{i=1,2, \ldots, k}
$$

defines a Cartier divisor on $\overline{T^{*} X}$. The corresponding Weil divisor is the spectral curve of the differential equation $d w=A w$ and denoted by

$$
C_{A} \subset \overline{T^{*} X}
$$


1.3. Arithmetic genus of spectral curve. We denote the divisor class of the spectral curve $C_{A}$ by the same notation. The arithmetic genus $g_{a}\left(C_{A}\right)$ of $C_{A}$ can be obtained by the genus formula

$$
g_{a}\left(C_{A}\right)=\frac{1}{2}\left(C_{A}, C_{A}+K_{\overline{T^{*} X}}\right)+1 .
$$

Our complex surface $\overline{T^{*} X}$ is a ruled surface of which the Neron-Severi group is well-understood. Thus standard argument enables us to examine the explicit value of $g_{a}\left(C_{A}\right)$, see V.2 in [11] and [10] for example.

Let us first determine the coefficients $a, b$ in the expression $C_{A}=a X_{0}+$ $b f \in \mathrm{NS} \overline{T^{*} X}$. Since the projection $\left.\pi\right|_{C_{A}}: C_{A} \rightarrow X$ is of degree $n$, we have

$$
\left(C_{A}, f\right)=n \text {. }
$$

Thus

$$
n=\left(C_{A}, f\right)=\left(a X_{0}+b f, f\right)=a .
$$

Next we note that $\left(X_{\infty}, X_{0}\right)=0$ and $\left(X_{\infty}, f\right)=1$. This shows that

$$
b=\left(n X_{0}+b f\right) X_{\infty}=\left(C_{A}, X_{\infty}\right)
$$

and we have

$$
C_{A}=n X_{0}+\left(C_{A}, X_{\infty}\right) f \in \mathrm{NS} \overline{T^{*} X} .
$$

Also note that

$$
K_{\overline{T^{*} X}}=-2 X_{0}+\left(2 g-2+\operatorname{deg} T^{*} X\right) f=-2 X_{0}+(4 g-4) f .
$$

Finally, the genus formula leads us to

$$
\begin{aligned}
g_{a}\left(C_{A}\right) & =\frac{1}{2}\left(n X_{0}+\left(C_{A}, X_{\infty}\right) f,(n-2) X_{0}+\left(\left(C_{A}, X_{\infty}\right)+4 g-4\right) f\right)+1 \\
& =\frac{1}{2}\left(n^{2}(2 g-2)+(2 n-2)\left(C_{A}, X_{\infty}\right)\right)+1 .
\end{aligned}
$$

\section{LOCAL FORMAL THEORY ON DIFFERENTIAL EQUATIONS}

Here we recall the Hukuhara-Turrittin-Levelt theory on local structure of differential equations and the notion of irregularity introduced by Komatsu 21] and Malgrange [24].

2.1. Differential modules over differential fields. First let us fix notation. Let $\mathbb{C} \llbracket z \rrbracket$ and $\mathbb{C}((z))$ denote the ring of formal power series and the field of formal Laurent series respectively. Similarly $\mathbb{C}\{z\}$ and $\mathbb{C}(\{z\})$ denote the ring of convergent power series and the field of convergent Laurent series. Let $\mathcal{P}:=\bigcup_{s \in \mathbb{Z}_{>0}} \mathbb{C}\left(\left(z^{\frac{1}{s}}\right)\right)$ be the field of Puiseux series. Also $\mathcal{P}^{\text {conv }}$ denote the field of convergent Puiseux series. Set $\mathcal{P}^{+}:=\bigcup_{s \in \mathbb{Z}_{>0}} \mathbb{C} \llbracket z^{\frac{1}{s}} \rrbracket$ $\mathcal{P}^{-}:=\bigcup_{s \in \mathbb{Z}_{>0}} z^{-\frac{1}{s}} \mathbb{C}\left[z^{-\frac{1}{s}}\right]$. Then we can decompose

$$
\mathcal{P}=\mathcal{P}^{-} \oplus \mathcal{P}^{+} .
$$

The order of $f(z)=\sum_{r \in \mathbb{Q}} a_{r} z^{r}$ is the number

$$
\operatorname{ord} f(z):=\underset{5}{\min }\left\{r \in \mathbb{Q} \mid a_{r} \neq 0\right\} \text {. }
$$


Similarly, the order of Puiseux series $G(z)=\sum_{r \in \mathbb{Q}} G_{r} z^{r} \in \mathcal{P} \otimes_{\mathbb{C}} M(n, \mathbb{C})$ with matrix coefficients is defined by ord $G(z):=\min \left\{r \in \mathbb{Q} \mid G_{r} \neq \mathbf{0}_{n}\right\}$ where $\mathbf{0}_{n}$ is the zero matrix of size $n$.

Let $\mathcal{K}$ be one of the following fields: $\mathbb{C}((z)), \mathbb{C}(\{z\}), \mathbb{C}\left(\left(z^{\frac{1}{s}}\right)\right), \mathbb{C}\left(\left\{z^{\frac{1}{s}}\right\}\right), \mathcal{P}$ and $\mathcal{P}^{\text {conv }}$. A differential module $M$ over $\mathcal{K}$ is a $\mathcal{K}$-module with the derivation $\nabla_{M} \in$ End $_{\mathbb{C}}(M)$ satisfying the Leibniz rule $\nabla_{M}(k m)=\frac{d}{d z} k \cdot m+k \cdot \nabla_{M} m$ for $k \in \mathcal{K}$ and $m \in M$. Suppose that $M$ is finite of rank $n$ over $\mathcal{K}$ and choose a basis $\mathbf{e}=\left\{e_{1}, e_{2}, \ldots, e_{n}\right\}$. Then the matrix $G={ }^{t}\left(g_{i, j}\right)_{1 \leq i, j \leq n}$ defined by

$$
\nabla_{M} e_{i}=\sum_{j=1}^{n} g_{i, j} e_{j}
$$

gives the matrix form of $\nabla_{M} \in \operatorname{End}_{\mathbb{C}}(M)$, that is

$$
\frac{d}{d z}-G \in \operatorname{End}_{\mathbb{C}}\left(\mathcal{K}^{\oplus n}\right) \text {. }
$$

We call $G$ the matrix of $\nabla_{M}$ with respect to e. Conversely, $G \in M(n, \mathcal{K})$ defines a differential module $M_{G}:=\mathcal{K}^{\oplus n}$ with the derivation $\nabla_{M_{G}}:=\frac{d}{d z}-G$.

For two matrices $G, G^{\prime}$ of $M$, there exists a base change matrix $X \in$ $\mathrm{GL}(n, \mathcal{K})$ and we have

$$
G^{\prime}=X G X^{-1}+\left(\frac{d}{d z} X\right) X^{-1}
$$

Let us recall some operations on finite differential modules. For differential modules $M$ and $M^{\prime}$, the direct product $M \oplus M^{\prime}$ is naturally defined as $\mathcal{K}$-modules equipped with the derivation

$$
\nabla_{M \oplus M^{\prime}}\left(m+m^{\prime}\right):=\nabla_{M} m+\nabla_{M^{\prime}} m^{\prime} \quad\left(m \in M, n^{\prime} \in M^{\prime}\right) .
$$

Also we can define the tensor product $M \otimes_{\mathcal{K}} M^{\prime}$ with the derivation

$$
\nabla_{M \otimes \mathcal{K} M^{\prime}}\left(m \otimes m^{\prime}\right):=\nabla_{M} m \otimes m^{\prime}+m \otimes \nabla_{M^{\prime}} m^{\prime} \quad\left(m \in M, n^{\prime} \in M^{\prime}\right) .
$$

The dual module of $M$ is $M^{*}:=\operatorname{Hom}_{\mathcal{K}}(M, \mathcal{K})$ with the derivation $\nabla_{M^{*}}$ satisfying the following. If $G$ is the matrix of $\nabla_{M}$ with respect to a basis $\mathbf{e}$, then $-{ }^{t} G$ is the matrix of $\nabla_{M^{*}}$ with respect to the dual basis $\mathbf{f}$ of $\mathbf{e}$.

The identification

$$
\operatorname{Hom}_{\mathcal{K}}\left(M, M^{\prime}\right) \cong M^{*} \otimes_{\mathcal{K}} M
$$

induces the differential module structure on $\operatorname{Hom}_{\mathcal{K}}\left(M, M^{\prime}\right)$.

2.2. Hukuhara-Turrittin-Levelt normal forms. We shall review the Hukuhara-Turrittin-Levelt theory which gives a formal classification of local differential equations. We use the notation

$$
\operatorname{diag}\left(A_{1}, A_{2}, \ldots, A_{k}\right)
$$

which stands for a block diagonal matrix with the diagonal entries $A_{i} \in$ $M\left(n_{i}, \mathcal{K}\right)$. Recall that the substitution $\xi: f(z) \mapsto f\left(e^{2 \pi i} z\right)$ for $f(z) \in \mathbb{C}\left(\left(z^{\frac{1}{s}}\right)\right)$ generates the Galois group

$$
\operatorname{Gal}\left(\mathbb{C}\left(\left(z^{\frac{1}{s}}\right)\right) / \mathbb{C}((z))\right) \cong \mu_{s}
$$

where $\mu_{s}$ is the cyclic group which consists of sth roots of 1 in $\mathbb{C}$. 
Definition 2.1 (HTL cell). Take $q(z) \in \mathcal{P}^{-}$and set $r=\min \left\{s \in \mathbb{Z}_{>0} \mid\right.$ $\left.q(z) \in z^{-\frac{1}{s}} \mathbb{C}\left[z^{-\frac{1}{s}}\right]\right\}$. Then the elementary Hukuhara-Turrittin-Levelt cell $E_{q(z), R}$ for the above $q(z)$ and $R \in M(n, \mathbb{C})$ is

$$
\begin{aligned}
& E_{q(z), R}:= \\
& \qquad \operatorname{diag}\left(q(z) I_{n}+R, \xi(q)(z) I_{n}+R, \ldots, \xi^{r-1}(q)(z) I_{n}+R\right) z^{-1} \\
& \quad \in M\left(r n, \mathbb{C}\left(\left(z^{\frac{1}{r}}\right)\right)\right)
\end{aligned}
$$

Here we call the integers $n$ and $r$ multiplicity and ramification index of $E_{q(z), R}$ respectively.

Definition 2.2 (HTL normal form). A Hukuhara-Turrittin-Levelt normal form is a matrix

$$
\operatorname{diag}\left(E_{q_{1}(z), R_{1}}, \ldots, E_{q_{m}(z), R_{m}}\right)
$$

with elementary HTL cells $E_{q_{i}(z), R}$ for $i=1,2, \ldots, m$ such that

$$
\operatorname{Gal}(\mathcal{P} / \mathbb{C}((z))) \cdot q_{i}(z) \cap \operatorname{Gal}(\mathcal{P} / \mathbb{C}((z))) \cdot q_{j}(z)=\emptyset, \quad \text { if } i \neq j .
$$

Theorem 2.3 (Hukuhara-Turrittin-Levelt, [14, [33, 23]). Let $M$ be a differential module over $\mathbb{C}(\{z\})$ of rank $n$ and $\widetilde{M}:=\mathbb{C}((z)) \otimes_{\mathbb{C}(\{z\})} M$ the formalization of $M$. Then there exists an HTL normal form

$$
\operatorname{diag}\left(E_{q_{1}(z), R_{1}}, \ldots, E_{q_{m}(z), R_{m}}\right)
$$

as a matrix of $\bar{M}:=\mathcal{P} \otimes_{\mathbb{C}((z))} \widetilde{M}$ with respect to a suitable basis. Furthermore, if two differential modules $M$ and $M^{\prime}$ over $\mathbb{C}(\{z\})$ share a same HTL normal form, then $\widetilde{M} \cong \widetilde{M^{\prime}}$.

The HTL normal form induces the following decomposition of $M$.

Theorem 2.4 (see (7.15) in [2] and COROLLARY 3.3 in [29]). We use the same notation as in Theorem [2.3. There exists a differential module $M_{E_{q_{i}(z), R_{i}}}$ over $\mathbb{C}(\{z\})$ whose HTL normal form is $E_{q_{i}(z), R_{i}}$ for each $i=$ $1,2, \ldots, m$ and we have a decomposition

$$
\widetilde{M} \cong \bigoplus_{i=1}^{m} \widetilde{M_{E_{q_{i}(z), R_{i}}}}
$$

as differential modules over $\mathbb{C}((z))$.

2.3. Komatsu-Malgrange irregularity. Let us recall that the index of a $\mathbb{C}$-linear endmorphism $\Phi$ is

$$
\chi(\Phi):=\operatorname{dim}_{\mathbb{C}} \operatorname{Ker} \Phi-\operatorname{dim}_{\mathbb{C}} \operatorname{Coker} \Phi .
$$

The Komatsu-Malgrange irregularity is an analytic invariant of local differential equations defined as follows.

Definition 2.5 (Komatsu-Malgrange irregularity). Let $M$ be a finite differential module over $\mathbb{C}(\{z\})$ and $\widetilde{M}:=\mathbb{C}((z)) \otimes_{\mathbb{C}(\{z\})} M$ its formalization.

Then the Komatsu-Malgrange irregularity of $M$ is

$$
\operatorname{Irr}(M):=\chi \underset{7}{\chi\left(\nabla_{\overparen{M}}\right)}-\chi\left(\nabla_{M}\right)
$$


If $M$ has the HTL-normal form

$$
\operatorname{diag}\left(E_{q_{1}(z), R_{1}}, \ldots, E_{q_{m}(z), R_{m}}\right),
$$

then it is known that the Komatsu-Malgrange irregularity is

$$
\operatorname{Irr}(M)=-\sum_{i=1}^{m} \sum_{j=0}^{r_{i}-1} \operatorname{ord} \xi^{j}\left(q_{i}\right)(z)=-\sum_{i=1}^{m} r_{i} \operatorname{ord} q_{i}(z) .
$$

Here $r_{i}$ are ramification indices of $E_{q_{i}(z), R_{i}}$ for $i=1,2, \ldots, m$.

\section{LOCAL COMPARISON: MilnOR FORMULA}

In this section we deal with a local differential module and define its characteristic polynomial with respect to a fixed basis. The zero locus of this characteristic polynomial may have a singularity at infinity which corresponds to the irregular singularity of the differential module. We shall compare these singularities and obtain a comparison formula between the irregularity of differential module and the Milnor number of the characteristic polynomial.

\subsection{Hukuhara-Turrittin-Levelt normal form and decomposition of characteristic polynomial.}

Definition 3.1 (characteristic polynomial). Let us consider a finite differential module $M$ over $\mathbb{C}(\{z\})$ of rank $n$. Fix a matrix $G \in M(n, \mathbb{C}(\{z\}))$ of $M$ with respect to a basis $\mathbf{e}$. Then the characteristic polynomial of $M$ with respect to $\mathbf{e}$ is

$$
\operatorname{det}\left(z^{\nu} y I_{n}-z^{\nu} G\right)=z^{n \nu} \operatorname{det}\left(y I_{n}-G\right) \in \mathbb{C}\{z\}[y] .
$$

Here $\nu \in \mathbb{Z}_{\geq 0}$ is the pole order of $G$.

The characteristic polynomial may have a singularity at $(y, z)=(\infty, 0)$. We shall see that the HTL normal form of $M$ have some information on the singularity.

Definition 3.2 (multiplicity free HTL normal form). An HTL normal form

$$
\operatorname{diag}\left(E_{q_{1}(z), R_{1}}, \ldots, E_{q_{m}(z), R_{m}}\right)
$$

is said to be multiplicity free when all HTL cells $E_{q_{i}(z), R_{i}}, i=1,2, \ldots, m$, are multiplicity one, namely, $R_{i} \in M(1, \mathbb{C})$ for all $i=1,2, \ldots, m$.

Proposition 3.3. Let $M$ be a differential module over $\mathbb{C}(\{z\})$ of rank $n$ and fix a matrix $G \in M(n, \mathbb{C}(\{z\}))$ of $M$.

Suppose that the $M$ has the multiplicity free HTL normal form

$$
\operatorname{diag}\left(E_{q_{1}(z), R_{1}}, \ldots, E_{q_{m}(z), R_{m}}\right) .
$$

Then the characteristic polynomial $z^{n \nu} \operatorname{det}\left(y I_{n}-G\right) \in \mathbb{C}\{z\}[y]$ decomposes as follows,

$$
z^{n \nu} \operatorname{det}\left(y I_{n}-G\right)=z^{n \nu} \prod_{i=1}^{m} \prod_{j=0}^{r_{i}-1}\left(y-\frac{\tilde{q}_{[i, j]}(z)}{z}\right) .
$$

Here $\tilde{q}_{[i, j]}(z) \in \mathcal{P}^{\text {conv }}$ satisfies that

$$
\operatorname{pr}^{-}\left(\tilde{q}_{[i, j]}(z)\right)=\xi^{j} q_{i}(z)
$$


for each $i=1,2, \ldots, m, j=0,1, \ldots, r_{i}-1$, and $\mathrm{pr}^{-}: \mathcal{P} \rightarrow \mathcal{P}^{-}$is the projection along the decomposition $\mathcal{P}=\mathcal{P}^{-} \oplus \mathcal{P}^{+}$.

Proof. For $q_{i}(z), i=1,2, \ldots, m$, define

$$
E_{q_{i}(z)}^{o}:=\operatorname{diag}\left(q_{i}(z), \xi\left(q_{i}\right)(z), \ldots, \xi^{r_{i}-1}\left(q_{i}\right)(z)\right) z^{-1} \in M\left(r_{i}, \mathbb{C}\left(\left(z^{\frac{1}{r_{i}}}\right)\right)\right) .
$$

Then the multiplicity free condition leads to

$$
\operatorname{diag}\left(E_{q_{1}(z), R_{1}}, \ldots, E_{q_{m}(z), R_{m}}\right) \equiv \operatorname{diag}\left(E_{q_{1}(z)}^{o}, \ldots, E_{q_{m}(z)}^{o}\right) \quad\left(\bmod z^{-1} \mathbb{C} \llbracket z^{\frac{1}{s}} \rrbracket\right) .
$$

Here $s:=\operatorname{lcm}\left\{r_{1}, r_{2}, \ldots, r_{m}\right\}$. Thus there exists $X \in \operatorname{GL}\left(n, \mathbb{C}\left(\left(z^{\frac{1}{s}}\right)\right)\right)$ such that

$$
X G X^{-1}+\left(\frac{d}{d z} X\right) X^{-1} \equiv \operatorname{diag}\left(E_{q_{1}(z)}^{o}, \ldots, E_{q_{m}(z)}^{o}\right) \quad\left(\bmod z^{-1} \mathbb{C} \llbracket z^{\frac{1}{s}} \rrbracket\right) .
$$

Since ord $\left(\frac{d}{d z} X\right) X^{-1} \geq-1$, we have

$$
X G X^{-1} \equiv \operatorname{diag}\left(E_{q_{1}(z)}^{o}, \ldots, E_{q_{m}(z)}^{o}\right) \quad\left(\bmod z^{-1} \mathbb{C} \llbracket z^{\frac{1}{s}} \rrbracket\right) .
$$

Note that all the entries $\xi^{i}\left(q_{j}\right)(z)$ in $\operatorname{diag}\left(E_{q_{1}(z)}^{o}, \ldots, E_{q_{m}(z)}^{o}\right)$ are mutually different. Thus applying the Lemma 3.4 below repeatedly, we can find $X^{\prime} \in$ $\operatorname{GL}\left(n, \mathbb{C}\left(\left(z^{\frac{1}{s}}\right)\right)\right)$ so that $X^{\prime} X G\left(X^{\prime} X\right)^{-1}$ is a diagonal matrix and

$$
X^{\prime} X G\left(X^{\prime} X\right)^{-1} \equiv \operatorname{diag}\left(E_{q_{1}(z)}^{o}, \ldots, E_{q_{m}(z)}^{o}\right) \quad\left(\bmod z^{-1} \mathbb{C} \llbracket z^{\frac{1}{s}} \rrbracket\right) .
$$

This leads us to the decomposition

$$
\operatorname{det}\left(y I_{n}-G\right)=\prod_{i=1}^{m} \prod_{j=0}^{r_{i}-1}\left(y-\frac{\tilde{q}_{[i, j]}(z)}{z}\right)
$$

with $\tilde{q}_{[i, j]}(z) \in \mathbb{C}\left(\left(z^{\frac{1}{s}}\right)\right)$ satisfying

$$
\tilde{q}_{[i, j]}(z) \equiv \xi^{j}\left(q_{i}\right)(z) \quad\left(\bmod \mathbb{C} \llbracket z^{\frac{1}{s}} \rrbracket\right)
$$

for each $i=1,2, \ldots, m, j=0,1, \ldots, r_{i}-1$. Since the field $\mathcal{P}^{\text {conv }}$ is algebraically closed, the equation (2) coincides with the decomposition in $\mathcal{P}^{\text {conv }}[y]$. Thus the formal Puiseux series $\tilde{q}_{[i, j]}(z)$ should be convergent power series.

The following lemma is just a slight modification of the standard and well-known argument in the local formal theory of differential equations, so called the splitting lemma, see Lemma 3 in the section 3.2 in 3 for example.

Lemma 3.4. Let us consider $A(t)=t^{r} \sum_{i=0}^{\infty} A_{i} t^{i} \in M(n, \mathbb{C}((t)))$ and suppose that $A_{0}=\operatorname{diag}\left(A_{0}^{11}, A_{0}^{22}\right) \in M(n, \mathbb{C})$ with $A_{0}^{j j} \in M\left(n_{j}, \mathbb{C}\right), j=1,2$, and the sets of eigenvalues of $A_{0}^{11}$ and $A_{0}^{22}$ respectively are disjoint. Then there exists

$$
T(z)=\left(\begin{array}{cc}
I_{n_{1}} & T_{12}(t) \\
T_{21}(z) & I_{n_{2}}
\end{array}\right), \quad T_{j k}(t)=\sum_{i=1}^{\infty} T_{i}^{j k} t^{i}, j, k=1,2
$$

such that

$$
T(t) A(t) T^{-1}(t)=\left(\begin{array}{cc}
B_{11}(t) & 0 \\
0 & B_{22}(t)
\end{array}\right)
$$

where $B_{j j}(t)=t^{r} \sum_{i=0}^{\infty} B_{i}^{j j} t^{i} \in M\left(n_{j}, \mathbb{C}((t))\right)$ with $B_{0}^{j j}=A_{0}^{j j}, j=1,2$. 
Proof. The proof is almost the same as that of the splitting lemma in the local theory of differential equations.

Let us write

$$
A(t)=\left(\begin{array}{ll}
A_{11}(t) & A_{12}(t) \\
A_{21}(t) & A_{22}(t)
\end{array}\right)
$$

where $A_{j k}(t)=t^{r} \sum_{i=0}^{\infty} A_{i}^{j k} t^{i} \in M\left(n_{j} \times n_{k}, \mathbb{C}((t))\right), j, k=1,2$. Then the equation

is equivalent to

$$
T(t) A(t)=\left(\begin{array}{cc}
B_{11}(t) & 0 \\
0 & B_{22}(t)
\end{array}\right) T(t)
$$

$$
\begin{aligned}
& B_{j j}(t)=A_{j j}(t)+T_{j k}(t) A_{k j}(t) \\
& A_{j k}(t)+T_{j k}(t) A_{k k}(t)=B_{j j}(t) T_{j k}(t)
\end{aligned}
$$

for $1 \leq j \neq k \leq 2$. Comparing the coefficients of the powers of $t$ on both sides, we have

$$
\begin{aligned}
T_{n}^{j k} A_{0}^{k k}-A_{0}^{j j} T_{n}^{j k}= & \sum_{\mu=1}^{n-1}\left(A_{n-\mu}^{j j} T_{\mu}^{j k}-T_{\mu}^{j k} A_{n-\mu}^{k k}\right) \\
& -\sum_{\nu=1}^{n-2} T_{\nu}^{j k} \sum_{\mu=1}^{n-\nu-1} A_{n-\mu-\nu}^{k j} T_{\mu}^{j k}+A_{n}^{j k}
\end{aligned}
$$

for $n \geq 1$. Recall that the equation

$$
T A_{0}^{22}-A_{0}^{11} T=C
$$

for a given $C \in M\left(n_{1} \times n_{2}, \mathbb{C}\right)$ has the unique solution $T \in M\left(n_{1} \times n_{2}, \mathbb{C}\right)$ since the sets of eigenvalues of $A_{0}^{11}$ and $A_{0}^{22}$ respectively are disjoint, see Lemma 24 of the section A.1 in [3] for example. Thus the above equations determine $T_{n}^{j k}, n=1,2, \ldots$, inductively.

Since the HTL normal form is multiplicity free, the decomposition in Theorem 2.4

$$
\widetilde{M} \cong \bigoplus_{i=1}^{m} \widetilde{M_{E_{q_{i}(z), R_{i}}}}
$$

is the irreducible decomposition. Correspondingly, the following proposition shows that the decomposition in Proposition 3.3 is the irreducible decomposition with the irreducible components

$$
\prod_{j=0}^{r_{i}-1}\left(y-\frac{\tilde{q}_{[i, j]}(z)}{z}\right) \in \mathbb{C}(\{z\})[y], \quad i=1,2, \ldots, m .
$$

Proposition 3.5. We use the same notation as in Proposition 3.3. The Galois orbit of $\tilde{q}_{[i, j]}(z) \in \mathcal{P}^{\text {conv }}$ is

$$
\begin{aligned}
\operatorname{Gal}(\mathcal{P} / \mathbb{C}((z))) \cdot \tilde{q}_{[i, j]}(z) & =\left\{\tilde{q}_{[i, 0]}(z), \tilde{q}_{[i, 1]}(z), \ldots, \tilde{q}_{\left[i, r_{i}-1\right]}(z)\right\} \\
& =\left\{\tilde{q}_{[i, 0]}(z), \xi\left(\tilde{q}_{[i, 0]}\right)(z), \ldots, \xi^{r_{i}-1}\left(\tilde{q}_{[i, 0]}\right)(z)\right\}
\end{aligned}
$$

for each $i=1,2, \ldots, m, j=0,1, \ldots, r_{i-1}$. In particular $\tilde{q}_{[i, j]}(z) \in \mathbb{C}\left(\left\{z^{\frac{1}{r_{i}}}\right\}\right)$. 
Proof. The decomposition

$$
\operatorname{det}\left(y I_{n}-G\right)=\prod_{i=1}^{m} \prod_{j=0}^{r_{i}-1}\left(y-\frac{\tilde{q}_{[i, j]}(z)}{z}\right) \in \mathbb{C}(\{z\})[y]
$$

tells us that

$$
\operatorname{Gal}(\mathcal{P} / \mathbb{C}((z))) \cdot \tilde{q}_{[i, j]}(z) \subset\left\{\tilde{q}_{[k, l]}(z) \mid k=1,2, \ldots, m, l=0,1, \ldots, r_{k}\right\} .
$$

Let $\mathrm{pr}^{-}: \mathcal{P} \rightarrow \mathcal{P}^{-}$be the projection along the decomposition $\mathcal{P}=\mathcal{P}^{-} \oplus \mathcal{P}^{+}$. Since $\mathrm{pr}^{-}$is compatible with the Galois action, we have

$$
\begin{aligned}
\operatorname{pr}^{-}\left(\operatorname{Gal}(\mathcal{P} / \mathbb{C}((z))) \cdot \tilde{q}_{[i, j]}(z)\right) & =\operatorname{Gal}(\mathcal{P} / \mathbb{C}((z))) \cdot \xi^{j}\left(q_{i}\right)(z) \\
& =\operatorname{Gal}(\mathcal{P} / \mathbb{C}((z))) \cdot q_{i}(z) .
\end{aligned}
$$

The restriction

$$
\widetilde{\mathrm{pr}}^{-}:=\left.\operatorname{pr}^{-}\right|_{\left\{\tilde{q}_{[k, l]}(z) \mid k=1,2, \ldots, m, l=0,1, \ldots, r_{k}\right\}}
$$

is a bijection onto $\left\{\xi^{l}\left(q_{k}\right)(z) \mid k=1,2, \ldots, m, l=0,1, \ldots, r_{k}\right\}$. Thus we have

$$
\begin{aligned}
\operatorname{Gal}(\mathcal{P} / \mathbb{C}((z))) \cdot \tilde{q}_{[i, j]}(z) & =\left(\widetilde{p r}^{-}\right)^{-1}\left(\operatorname{Gal}(\mathcal{P} / \mathbb{C}((z))) \cdot q_{i}(z)\right) \\
& =\left\{\tilde{q}_{[i, 0]}(z), \tilde{q}_{[i, 1]}(z), \ldots, \tilde{q}_{\left[i, r_{i}-1\right]}(z)\right\} \\
& =\left\{\tilde{q}_{[i, 0]}(z), \xi\left(\tilde{q}_{[i, 0]}\right)(z), \ldots, \xi^{r_{i}-1}\left(\tilde{q}_{[i, 0]}\right)(z)\right\} .
\end{aligned}
$$

3.2. Milnor formula. By Proposition 3.5, the decomposition in Proposition 3.3 can be rewritten as follows,

$$
\begin{aligned}
z^{n \nu} \operatorname{det}\left(y I_{n}-G\right) & =z^{n \nu} \prod_{i=1}^{m} \prod_{j=0}^{r_{i}-1}\left(y-\frac{\xi^{j}\left(\tilde{q}_{i}\right)(z)}{z}\right) \\
& =\prod_{i=1}^{m} \prod_{j=0}^{r_{i}-1} z^{\nu_{i}}\left(y-\frac{\xi^{j}\left(\tilde{q}_{i}\right)(z)}{z}\right),
\end{aligned}
$$

where $\tilde{q}_{i}(z) \in \mathbb{C}\left(\left\{z^{\frac{1}{r_{i}}}\right\}\right)$ satisfy $\operatorname{pr}^{-}\left(\tilde{q}_{i}(z)\right)=q_{i}(z)$ and we set $\nu_{i}:=r_{i}$. $\max \left\{0,-\operatorname{ord} \frac{q_{i}(z)}{z}\right\}$ for $i=1,2, \ldots, m$. Moreover this is the irreducible decomposition with the irreducible components

$$
z^{\nu_{i}} \prod_{j=0}^{r_{i}-1}\left(y-\frac{\xi^{j}\left(\tilde{q}_{i}\right)(z)}{z}\right) \in \mathbb{C}\{z\}[y] .
$$

We now investigate the singularity of the zero locus of each irreducible components at $(y, z)=(\infty, 0)$. To be more precise, let us put $y=\frac{\eta}{\zeta}$ and consider the homogenized polynomial

$$
z^{\nu_{i}} \prod_{j=0}^{r_{i}-1}\left(\eta-\frac{\xi^{j}\left(\tilde{q}_{i}\right)(z)}{z} \zeta\right) .
$$


The restriction to $\eta=1$ gives

$$
z^{\nu_{i}} \prod_{j=0}^{r_{i}-1}\left(1-\frac{\xi^{j}\left(\tilde{q}_{i}\right)(z)}{z} \zeta\right)=z^{\nu_{i}} \prod_{j=0}^{r_{i}-1}\left(-\frac{\xi^{j}\left(\tilde{q}_{i}\right)(z)}{z}\right) \cdot \prod_{j=0}^{r_{i}-1}\left(\zeta-\frac{z}{\xi^{j}\left(\tilde{q}_{i}\right)(z)}\right) .
$$

Suppose that ord $\frac{\tilde{q_{i}}(z)}{z}<0$. Then

$$
\left.z^{\nu_{i}} \prod_{j=0}^{r_{i}-1}\left(-\frac{\xi^{j}\left(\tilde{q}_{i}\right)(z)}{z}\right)\right|_{z=0} \neq 0 \quad \text { and } \quad \frac{z}{\xi^{j}\left(\tilde{q}_{i}\right)(z)} \in \mathbb{C}\left(\left\{z^{\frac{1}{r_{i}}}\right\}\right) \cap \mathbb{C} \llbracket z^{\frac{1}{r_{i}}} \rrbracket,
$$

and the zero locus of

$$
\prod_{j=0}^{r_{i}-1}\left(\zeta-\frac{z}{\xi^{j}\left(\tilde{q}_{i}\right)(z)}\right)
$$

defines the plane curve germ $C_{\tilde{q}_{i}}$ at $(\zeta, z)=(0,0)$ where $\zeta:=\frac{1}{y}$.

Let us set

$$
-\frac{p_{i}}{r_{i}}:=\text { ord } q_{i}(z)
$$

with relatively prime integers $p_{i}$ and $r_{i}$ for $i=1,2, \ldots, m$.

Proposition 3.6. Let us fix $i \neq j \in\{1,2, \ldots, m\}$ such that ord $\frac{\tilde{q}_{i}(z)}{z}<0$ and ord $\frac{\tilde{q_{j}}(z)}{z}<0$. Then the intersection number of $C_{\tilde{q}_{i}}$ and $C_{\tilde{q}_{j}}$ is

$$
\left(C_{\tilde{q}_{i}}, C_{\tilde{q}_{j}}\right)=p_{i} r_{j}+p_{j} r_{i}+r_{i} r_{j}-\operatorname{Irr}\left(\operatorname{Hom}_{\mathbb{C}(\{z\})}\left(M_{E_{q_{i}, R_{i}}}, M_{E_{q_{j}, R_{j}}}\right)\right) .
$$

Proof. Since the plane curve germ $C_{\tilde{q}_{i}}$ is parametrized by $z(t)=t^{r_{i}}, \zeta(t)=$ $\frac{t^{r_{i}}}{\tilde{q}_{i}\left(t^{r_{i}}\right)}$ and the germ $C_{\tilde{q}_{j}}$ is defined by $\prod_{k=1}^{r_{j}}\left(\zeta-\frac{z}{\xi^{k}\left(\tilde{q}_{j}\right)(z)}\right)=0$. Then the intersection number of them is computed as follows, see 1.2 in [34] for example.

$$
\begin{aligned}
\left(C_{\tilde{q_{i}}}, C_{\tilde{q}_{j}}\right) & =\operatorname{ord}_{t} \prod_{k=1}^{r_{j}}\left(\zeta(t)-\frac{t^{r_{i}}}{\xi^{k}\left(\tilde{q}_{j}\right)\left(t^{r_{i}}\right)}\right) \\
& =r_{i} \operatorname{ord}_{z} \prod_{k=1}^{r_{j}}\left(\frac{z}{\tilde{q}_{i}(z)}-\frac{z}{\xi^{k}\left(\tilde{q_{j}}\right)(z)}\right) \\
& =r_{i} \operatorname{ord}_{z} \prod_{k=1}^{r_{j}}\left(\frac{1}{\tilde{q}_{i}(z)}-\frac{1}{\xi^{k}\left(\tilde{q_{j}}\right)(z)}\right)+r_{i} r_{j} \\
& =r_{i} \operatorname{ord} z \prod_{k=1}^{r_{j}}\left(\frac{\xi^{k}\left(\tilde{q_{j}}\right)(z)-\tilde{q}_{i}(z)}{\tilde{q}_{i}(z) \xi^{k}\left(\tilde{q}_{j}\right)(z)}\right)+r_{i} r_{j} \\
& =r_{i} \operatorname{ord}_{z} \prod_{k=1}^{r_{j}}\left(\xi^{k}\left(\tilde{q}_{j}\right)(z)-\tilde{q}_{i}(z)\right)+p_{i} r_{j}+p_{j} r_{i}+r_{i} r_{j} \\
& =p_{i} r_{j}+p_{j} r_{i}+r_{i} r_{j}-\operatorname{Irr}\left(\operatorname{Hom}_{\mathbb{C}(\{z\})}\left(M_{E_{q_{i}, R_{i}}}, M_{E_{q_{j}, R_{j}}}\right)\right) .
\end{aligned}
$$

Proposition 3.7. Let us fix an $i \in\{1,2, \ldots, m\}$ and suppose that ord $\frac{\tilde{q}_{i}(z)}{z}<$ 0 . Then the Milnor number of the germ $C_{\tilde{q}_{i}}$ is

$$
\mu\left(C_{\tilde{q}_{i}}\right)=\left(2 p_{i}+r_{i}-1\right)\left(r_{i}-1\right)-\operatorname{Irr}\left(\operatorname{End}_{\mathbb{C}(\{z\})}\left(M_{E_{q_{i}, R_{i}}}\right)\right) .
$$


Proof. Set $F_{i}(\zeta, z):=\prod_{k=1}^{r_{i}}\left(\zeta-\frac{z}{\xi^{k}\left(\tilde{q_{i}}\right)(z)}\right)$. Then the germ $C_{\tilde{q}_{i}}$ is defined by $F_{i}=0$. Then the Milnor number can be obtained by

$$
\mu\left(C_{\tilde{q}_{i}}\right)=\left(F_{i}, \frac{\partial}{\partial \zeta} F_{i}\right)+1-\left(F_{i}, z\right) .
$$

We refer to COROLLARY 7.16 and THEOREM 7.18 in [12] for this fact.

$$
\begin{aligned}
& \text { If we note that } \frac{\partial}{\partial \zeta} F_{i}=\sum_{k=1}^{r_{i}} \prod_{\substack{1 \leq l \leq r_{i} \\
l \neq k}}\left(\zeta-\frac{z}{\xi^{l}\left(\tilde{q}_{i}\right)(z)}\right) \text {, then } \\
& \qquad \begin{array}{l}
\left(F_{i}, \frac{\partial}{\partial \zeta} F_{i}\right)=\operatorname{ord}_{z} \prod_{\substack{1 \leq l, k \leq r_{i} \\
l \neq k}}\left(\frac{z}{\xi^{k}\left(\tilde{q}_{i}\right)(z)}-\frac{z}{\xi^{l}\left(\tilde{q}_{i}\right)(z)}\right) \\
\quad=\operatorname{ord}_{z} \prod_{\substack{1 \leq l, k \leq r_{i} \\
l \neq k}}\left(\xi^{l}\left(\tilde{q}_{i}\right)(z)-\xi^{k}\left(\tilde{q}_{i}\right)(z)\right)+r_{i}\left(r_{i}-1\right)+2 p_{i}\left(r_{i}-1\right) \\
=-\operatorname{Irr}\left(\operatorname{End}_{\mathbb{C}(\{z\})}\left(M_{E_{q_{i}, R_{i}}}\right)\right)+r_{i}\left(r_{i}-1\right)+2 p_{i}\left(r_{i}-1\right) .
\end{array}
\end{aligned}
$$

Also we have $\left(F_{j}, z\right)=r_{i}$. Thus combining these equations, we have

$$
\begin{aligned}
\mu\left(C_{\tilde{q}_{i}}\right) & =\left(2 p_{i}+r_{i}\right)\left(r_{i}-1\right)-\operatorname{Irr}\left(\operatorname{End}_{\mathbb{C}(\{z\})}\left(M_{E_{q_{i}, R_{i}}}\right)\right)+1-r_{i} \\
& =\left(2 p_{i}+r_{i}-1\right)\left(r_{i}-1\right)-\operatorname{Irr}\left(\operatorname{End}_{\mathbb{C}(\{z\})}\left(M_{E_{q_{i}, R_{i}}}\right)\right) .
\end{aligned}
$$

Now we compute the Milnor number of the zero locus of the characteristic polynomial $z^{n \nu} \operatorname{det}\left(y I_{n}-G\right)$ at $(y, z)=(\infty, 0)$ as follows. Let us suppose that $M$ has a singularity at $z=0$. Then $G$ has a pole at $z=0$ and the zero locus of the homogenization of the characteristic polynomial

$$
z^{n \nu} \zeta^{n} \operatorname{det}\left(\frac{\eta}{\zeta} I_{n}-G\right)
$$

pass through the point $([\zeta: \eta], z)=([0: 1], 0)$. Let us denote the zero locus by $C_{G}$ and the Milnor number of $C_{G}$ at $([\zeta: \eta], z)=([0: 1], 0)$ by $\mu\left(C_{G}\right)_{\infty}$.

Theorem 3.8 (Milnor formula). Let us take a differential module $M$ over $\mathbb{C}(\{z\})$ of rank $n$ and a matrix $G$ of $M$ as in Proposition [3.3. Suppose that $M$ has a singularity at $z=0$. Then the Milnor number of $C_{G}$ at $([\zeta: \eta], z)=([0: 1], 0)$ is

$$
\mu\left(C_{G}\right)_{\infty}=-n^{2}-\operatorname{Irr}\left(\operatorname{End}_{\mathbb{C}(\{z\})}(M)\right)+2(n-1)\left(C_{G}, \zeta\right)_{\infty}+\left(m-r_{C_{G}}\right)+1 .
$$

Here $r_{C_{G}}$ is the number of branches of the germ of $C_{G}$ at $([\zeta: \eta], z)=([0$ : $1], 0)$ and $\left(C_{G}, \zeta\right)_{\infty}$ is the intersection number of $C_{G}$ and $\zeta$ at $([\zeta: \eta], z)=$ $([0: 1], 0)$.

Proof. First we assume that ord $\frac{\tilde{q}_{i}(z)}{z}<0$ for all $i=1,2, \ldots, m$. Then the decomposition in Proposition 3.3 shows that the homogenized characteristic 
polynomial

$z^{n \nu} \zeta^{n} \operatorname{det}\left(\frac{\eta}{\zeta} I_{n}-G\right)=z^{n \nu} \prod_{i=1}^{m}\left(\prod_{j=0}^{r_{i}-1}\left(-\frac{\xi^{j}\left(\tilde{q}_{i}\right)(z)}{z}\right) \cdot \prod_{j=0}^{r_{i}-1}\left(\zeta-\frac{z}{\xi^{j}\left(\tilde{q}_{i}\right)(z)} \eta\right)\right)$

defines a reduced plane curve germ $C_{G}$ at $([\zeta: \eta], z)=([0: 1], 0)$ with branches $C_{\tilde{q}_{i}}, i=1,2, \ldots, m$. Then by Propositions [3.6] and 3.7, the Milnor number of $C_{G}$ is

$$
\begin{aligned}
\mu\left(C_{G}\right)_{\infty}= & \sum_{i=1}^{m} \mu\left(C_{\tilde{q}_{i}}\right)+2 \sum_{1 \leq j<k \leq m}\left(C_{\tilde{q_{j}}}, C_{\tilde{q}_{k}}\right)-r_{C_{G}}+1 \\
= & -\sum_{1 \leq i, j \leq m} \operatorname{Irr}\left(\operatorname{Hom}_{\mathbb{C}(\{z\})}\left(M_{E_{q_{i}(z), R_{i}}}, M_{E_{q_{j}(z), R_{j}}}\right)\right) \\
& +\sum_{i=1}^{m}\left(2 p_{i}+r_{i}-1\right)\left(r_{i}-1\right)+2 \sum_{1 \leq j<k \leq m}\left(p_{j} r_{k}+p_{k} r_{j}+r_{j} r_{k}\right) \\
& -m+1 \\
= & -\operatorname{Irr}\left(\operatorname{End}_{\mathbb{C}(\{z\})}(M)\right)+\sum_{i=1}^{m}\left(2 p_{i} r_{i}+r_{i} r_{i}-2\left(p_{i}+r_{i}\right)\right)+m \\
& +2 \sum_{1 \leq j<k \leq m}\left(p_{j} r_{k}+p_{k} r_{j}+r_{j} r_{k}\right)-m+1 \\
= & -\operatorname{Irr}\left(\operatorname{End}_{\mathbb{C}(\{z\})}(M)\right)+2 \sum_{i=1}^{m} p_{i} \sum_{i=1}^{m} r_{i}+\sum_{i=1}^{m} r_{i} \sum_{i=1}^{m} r_{i} \\
& -2 \sum_{i=1}^{m}\left(p_{i}+r_{i}\right)+1 .
\end{aligned}
$$

Now let us note that $n=\sum_{i=1}^{m} r_{i}$ and

$$
\begin{aligned}
\left(C_{G}, \zeta\right) & =\sum_{i=1}^{m}\left(F_{i}, \zeta\right)=\sum_{i=1}^{m} \operatorname{ord}{ }_{t} \frac{t^{r_{i}}}{\tilde{q}_{i}\left(t^{r_{i}}\right)} \\
& =\sum_{i=1}^{m}\left(p_{i}+r_{i}\right) .
\end{aligned}
$$

Then we have

$$
\begin{aligned}
\mu\left(C_{G}\right)_{\infty}= & -\operatorname{Irr}\left(\operatorname{End}_{\mathbb{C}(\{z\})}(M)\right)+2 \sum_{i=1}^{m}\left(p_{i}+r_{i}-r_{i}\right) n+n^{2} \\
& -2 \sum_{i=1}^{m}\left(p_{i}+r_{i}\right)+1 \\
= & -\operatorname{Irr}\left(\operatorname{End}_{\mathbb{C}(\{z\})}(M)\right)+2(n-1) \sum_{i=1}^{m}\left(p_{i}+r_{i}\right)-n^{2}+1 \\
= & -n^{2}-\operatorname{Irr}\left(\operatorname{End}_{\mathbb{C}(\{z\})}(M)\right)+2(n-1)\left(C_{G}, \zeta\right)+1 .
\end{aligned}
$$


On the other hand, let us assume that there exists $i \in\{1,2, \ldots, m\}$ such that ord $\frac{\tilde{q}_{i}(z)}{z} \geq 0$. Then $\operatorname{pr}^{-}\left(\tilde{q}_{i}(z)\right)=q_{i}(z)$ must be 0 and ord $\frac{\tilde{q_{j}}(z)}{z}<0$ for the other $j \in\{1,2, \ldots, m\} \backslash\{i\}$ because $q_{j}(z) \neq 0$ by the definition of HTL normal forms. We may put $i=m$ by permuting the indices if necessary.

Let us note that $m \geq 2$ in this case. If $m=1$, then $\frac{\tilde{q}_{m}(z)}{z}=G$. Hence $G$ has no pole at $z=0$ and $M$ has no singularity at $z=0$.

In a way similar to the above argument, we can show that

$$
\begin{aligned}
\mu\left(C_{G}\right)_{\infty}= & \sum_{i=1}^{m-1} \mu\left(C_{\tilde{q}_{i}}\right)+2 \sum_{1 \leq j<k \leq m-1}\left(C_{\tilde{q}_{j}}, C_{\tilde{q}_{k}}\right)-r_{C_{G}}+1 \\
= & -\sum_{1 \leq i, j \leq m-1} \operatorname{Irr}\left(\operatorname{Hom}_{\mathbb{C}(\{z\})}\left(M_{E_{q_{i}(z), R_{i}}}, M_{E_{q_{j}(z), R_{j}}}\right)\right) \\
& +2 \sum_{i=1}^{m-1} p_{i} \sum_{i=1}^{m-1} r_{i}+\sum_{i=1}^{m-1} r_{i} \sum_{i=1}^{m-1} r_{i}-2 \sum_{i=1}^{m-1}\left(p_{i}+r_{i}\right)+1 .
\end{aligned}
$$

Now let us notice that

$$
\begin{aligned}
\operatorname{Irr}\left(\operatorname{End}_{\mathbb{C}(\{z\})}(M)\right)= & \sum_{1 \leq i, j \leq m-1} \operatorname{Irr}\left(\operatorname{Hom}_{\mathbb{C}(\{z\})}\left(M_{E_{q_{i}(z), R_{i}}}, M_{E_{q_{j}(z), R_{j}}}\right)\right) \\
& +2 \sum_{i=1}^{m-1} \operatorname{Irr}\left(\operatorname{Hom}_{\mathbb{C}(\{z\})}\left(M_{E_{q_{m}(z), R_{m}}}, M_{E_{q_{i}(z), R_{i}}}\right)\right) \\
& +\operatorname{Irr}\left(\operatorname{End}_{\mathbb{C}(\{z\})}\left(M_{E_{q_{m}(z), R_{m}}}\right)\right) \\
= & \sum_{1 \leq i, j \leq m-1} \operatorname{Irr}\left(\operatorname{Hom}_{\mathbb{C}(\{z\})}\left(M_{E_{q_{i}(z), R_{i}}}, M_{E_{q_{j}(z), R_{j}}}\right)\right) \\
& +2 \sum_{i=1}^{m-1} p_{i}, \\
\sum_{i=1}^{m} r_{i} \sum_{i=1}^{m} r_{i}= & \sum_{i=1}^{m-1} r_{i} \sum_{i=1}^{m-1} r_{i}+2 \sum_{i=1}^{m-1} r_{i}+1 \\
= & \sum_{i=1}^{m-1} r_{i} \sum_{i=1}^{m-1} r_{i}+2(n-1)+1, \\
\sum_{i=1}^{m}\left(p_{i}+r_{i}\right)= & \sum_{i=1}^{m-1}\left(p_{i}+r_{i}\right)+1, \\
\left(C_{G}, \zeta\right)_{\infty}= & \sum_{i=1}^{m-1}\left(p_{i}+r_{i}\right)=\sum_{i=1}^{m}\left(p_{i}+r_{i}\right)-1,
\end{aligned}
$$


where we use the fact $p_{m}=0$ and $r_{m}=1$. Then it follows that

$$
\begin{aligned}
\mu\left(C_{G}\right)_{\infty}= & -\operatorname{Irr}\left(\operatorname{End}_{\mathbb{C}(\{z\})}(M)+2 \sum_{i=1}^{m} p_{i} \sum_{i=1}^{m} r_{i}+\sum_{i=1}^{m} r_{i} \sum_{i=1}^{m} r_{i}\right. \\
& -2 \sum_{i=1}^{m}\left(p_{i}+r_{i}\right)-2(n-1)+2 \\
= & -n^{2}-\operatorname{Irr}\left(\operatorname{End}_{\mathbb{C}(\{z\})}(M)\right)+2(n-1)\left(C_{G}, \zeta\right)_{\infty}+2 \\
= & -n^{2}-\operatorname{Irr}\left(\operatorname{End}_{\mathbb{C}(\{z\})}(M)\right)+2(n-1)\left(C_{G}, \zeta\right)_{\infty}+\left(m-r_{C_{G}}\right)+1 .
\end{aligned}
$$

3.3. Differential modules with regular semisimple matrices over $\mathbb{C} \llbracket z \rrbracket$. In order to decompose the characteristic polynomial in accordance with the HTL normal form of the corresponding differential module, we have assumed the multiplicity free condition in Proposition 3.3. However if we consider a differential module with regular singularity, this module should be just rank 1 under this condition. Thus we now discuss another condition which we call regular semisimplicity over $\mathbb{C} \llbracket z \rrbracket$ and see that the previous argument is also valid under this condition.

Definition 3.9. Let us consider a differential module $M$ over $\mathbb{C}(\{z\})$ of rank $n$ with a matrix $G$. If there exists $X \in \mathrm{GL}(n, \mathbb{C} \llbracket z \rrbracket)$ such that

$$
X G X^{-1}+\left(\frac{d}{d z} X\right) X^{-1}=\operatorname{diag}\left(q_{1}(z), q_{2}(z), \ldots, q_{n}(z)\right) z^{-1}
$$

with mutually different polynomials $q_{i}(z) \in \mathbb{C}\left[z^{-1}\right]$ of $z^{-1}, i=1,2, \ldots, n$, then we say that $G$ is regular semisimple over $\mathbb{C} \llbracket z \rrbracket$ with the HTL normal form $\operatorname{diag}\left(q_{1}(z), q_{2}(z), \ldots, q_{n}(z)\right) z^{-1}$.

Remark 3.10. Let be $M$ a differential module over $\mathbb{C}(\{z\})$ with a matrix $G$. Then in papers [15] and [6], it is assumed that the leading coefficient of $G$ is diagonalizable with distinct eigenvalues if the pole order of $G$ at $z=0$ is greater than 1 , or diagonalizable with distinct eigenvalues $\bmod \mathbb{Z}$ if $G$ has a simple pole at $z=0$, see equation (1.3) in [15] and DEFINITION 2.2 in [6]. Then we can see that $G$ is regular semisimple under this condition.

Proposition 3.11. Let $M$ be a differential module over $\mathbb{C}(\{z\})$ of rank $n$ and fix a matrix $G \in M(n, \mathbb{C}(\{z\}))$ of $M$. form

Suppose that $G$ is regular semisimple over $\mathbb{C} \llbracket z \rrbracket$ with the $H T L$ normal

$$
\operatorname{diag}\left(q_{1}(z), q_{2}(z), \ldots, q_{n}(z)\right) z^{-1} .
$$

Then the characteristic polynomial $z^{n \nu} \operatorname{det}\left(y I_{n}-G\right) \in \mathbb{C}\{z\}[y]$ decomposes as follows,

$$
z^{n \nu} \operatorname{det}\left(y I_{n}-G\right)=z^{n \nu} \prod_{i=1}\left(y-\frac{\tilde{q}_{i}(z)}{z}\right) .
$$

Here $\tilde{q}_{i}(z) \in \mathbb{C}(\{z\})$ satisfies that

$$
\operatorname{pr}^{\leq 0}\left(\tilde{q}_{i}(z)\right)=q_{i}(z)
$$


for each $i=1,2, \ldots, n$, and $\operatorname{pr}^{\leq 0}: \mathbb{C}(\{z\}) \rightarrow \mathbb{C}\left[z^{-1}\right]$ is the projection along the decomposition $\mathbb{C}((z))=\mathbb{C}\left[z^{-1}\right] \oplus z \mathbb{C} \llbracket z \rrbracket$.

Proof. By the assumption, there exists $X \in \mathrm{GL}(n, \mathbb{C} \llbracket z \rrbracket)$ such that

$$
X G X^{-1}+\left(\frac{d}{d z} X\right) X^{-1}=\operatorname{diag}\left(q_{1}(z), q_{2}(z), \ldots, q_{n}(z)\right) z^{-1} .
$$

Since ord $\left(\frac{d}{d z} X\right) X^{-1} \geq 0$, it follows that

$$
X G X^{-1} \equiv \operatorname{diag}\left(q_{1}(z), q_{2}(z), \ldots, q_{n}(z)\right) z^{-1} \quad(\bmod \mathbb{C} \llbracket z \rrbracket) .
$$

The regular semisimplicity assures that all $q_{i}(z)$ are mutually different polynomials of $z^{-1}$. Thus the result follows from same argument in Proposition 3.3 .

The argument in Propositions 3.6, 3.7 and Theorem 3.8 is valid without any change even for this case. Thus the Milnor formula as we saw in Theorem 3.8 holds for this regular semisimple case.

Theorem 3.12. Let us take a differential module $M$ over $\mathbb{C}(\{z\})$ of rank $n$ and a matrix $G$ of $M$ as in Proposition [3.11. Suppose that $M$ has a singularity at $z=0$. Then the Milnor number of $C_{G}$ at $([\zeta: \eta], z)=([0:$ 1],0) is

$$
\mu\left(C_{G}\right)_{\infty}=-n^{2}-\operatorname{Irr}\left(\operatorname{End}_{\mathbb{C}(\{z\})}(M)\right)+2(n-1)\left(C_{G}, \zeta\right)_{\infty}+\left(n-r_{C_{G}}\right)+1 .
$$

\section{Global Comparison: Euler characteristics}

In the previous section, we have obtained a comparison formula of local singularities of a differential module and its characteristic polynomial. This local comparison implies the following coincidence of the global invariants, namely we shall show the matching of the index of rigidity of a differential equation and the Euler characteristic of the corresponding spectral curve.

We now come back to the differential equation

$$
d w=A w, \quad A \in M\left(n, \Omega_{X}(* D)(X)\right),
$$

on the Riemann surface $X$. We use the same notation as in Section 1 . Recall that $D=\left\{a_{1}, a_{2}, \ldots, a_{p}\right\}$ is the set of poles of $A$. This equation defines an algebraic connection $\nabla_{A}:=d-A$ on the trivial algebraic vector bundle

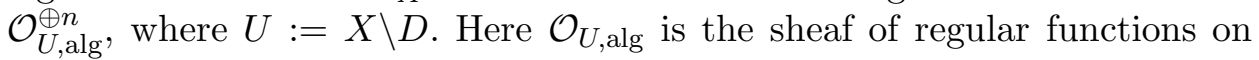
the Zariski open subset $U \subset X$.

Let $\nabla_{A}^{*}$ be the dual connection of $\nabla_{A}$, i.e., the dual bundle $\left(\mathcal{O}_{U, \text { alg }}^{\oplus n}\right)^{*}$ with the connection

$$
\nabla_{A}^{*}(\phi)(s)=-\phi\left(\nabla_{A} s\right)
$$

where $\phi$ and $s$ are sections of $\left(\mathcal{O}_{U, \text { alg }}^{\oplus n}\right)^{*}$ and $\mathcal{O}_{U, \text { alg }}^{\oplus n}$ respectively.

Further define the endomorphism connection as the tensor product,

$$
\mathcal{E} \operatorname{nd}\left(\nabla_{A}\right):=\nabla_{A}^{*} \otimes \nabla_{A} .
$$

Definition 4.1 (index of rigidity, Katz [20]). The index of rigidity of $\nabla_{A}$ is the Euler characteristic

$$
\operatorname{rig}\left(\nabla_{A}\right)=\sum_{i=0}^{2}(-1)^{i} \operatorname{dim}_{\mathbb{C}} H_{\mathrm{dR}}^{i}\left(X, j_{!_{*}}\left(\mathcal{E} \operatorname{nd} \nabla_{A}\right)\right) .
$$


Here $j_{\text {!* }}\left(\mathcal{E}\right.$ nd $\left.\nabla_{A}\right)$ is the middle extension by the embedding $j: U \hookrightarrow X$ of $\mathcal{E} \mathrm{nd} \nabla_{A}$ and $H_{\mathrm{dR}}^{*}\left(X, j_{!_{*}}\left(\mathcal{E} \mathrm{nd} \nabla_{A}\right)\right)$ are hypercohomology groups of the algebraic de Rham complex of $j_{! *}\left(\mathcal{E}\right.$ nd $\left.\nabla_{A}\right)$, see II.6 in [8] and 2.9 in [18] for more detailed treatment.

The Euler-Poincare formula by Deligne, Gabber and Katz, see THEOREM 2.9.9 in [18, gives a decomposition of $\operatorname{rig}\left(\nabla_{A}\right)$ into a sum of local invariants as follows,

$$
\operatorname{rig}\left(\nabla_{A}\right)=(2-2 g) \operatorname{rank}\left(\mathcal{E} \operatorname{nd}\left(\nabla_{A}\right)\right)-\sum_{a \in D} \delta\left(\operatorname{End}_{\mathbb{C}\left(\left\{z_{a}\right\}\right)}\left(M_{A_{a}}\right)\right)
$$

where

$$
\begin{aligned}
\delta\left(\operatorname{End}_{\mathbb{C}(\{z\})}\left(M_{A_{a}}\right)\right):= & \operatorname{rank} \operatorname{End}_{\mathbb{C}\left(\left\{z_{a}\right\}\right)}\left(M_{A_{a}}\right)+\operatorname{Irr}\left(\operatorname{End}_{\mathbb{C}\left(\left\{z_{a}\right\}\right)}\left(M_{A_{a}}\right)\right) \\
& -\operatorname{dim}_{\mathbb{C}} \operatorname{Hom}_{\mathbb{C}\left(\left(z_{a}\right)\right)}\left(\operatorname{End}_{\mathbb{C}\left(\left(z_{a}\right)\right)}\left(\widetilde{M_{A_{a}}}\right), \mathbb{C}\left(\left(z_{a}\right)\right)\right)^{\text {hor }}
\end{aligned}
$$

and $M^{\text {hor }}:=\left\{m \in M \mid \nabla_{M} m=0\right\}$ for a differential module $M$.

Take $a \in D$ and choose a local coordinate $\left(U_{i}, z_{i}\right)$ containing $a \in U_{i}$. Then we can write $A=A_{i} d z_{i}, A_{i} \in M\left(n, \mathbb{C}\left(z_{i}\right)\right)$ on $U_{i}$. Let us put $z_{a}:=z_{i}-$ $z_{i}(a)$. Then power series expansion of $A_{i}$ defines $A_{a} \in M\left(n, \mathbb{C}\left(\left\{z_{a}\right\}\right)\right)$ and a differential module $M_{A_{a}}:=\mathbb{C}\left(\left\{z_{a}\right\}\right)^{\oplus n}$ with the derivation $\nabla_{M_{A_{a}}}:=\frac{d}{d z_{a}}-A_{a}$. Let us denote the point $\left(\left[\zeta_{i}: \eta_{i}\right], z_{i}\right)=([0: 1], a)$ by $\infty_{a}$.

The following assumption enables us to apply the results in Section 3.1 to the connection $\nabla_{A}$.

Assumption 4.2. For each $a \in D$, the HTL normal form of $M_{A_{a}}$ is multiplicity free or $A_{a}$ is regular semisimple over $\mathbb{C} \llbracket z_{a} \rrbracket$.

Theorem 4.3. Under Assumption 4.2 we have the following. For each $a \in D$ the Milnor number of $C_{A}$ at $\infty_{a}$ is

$$
\mu\left(C_{A}\right)_{\infty_{a}}=-\delta\left(\operatorname{End}_{\mathbb{C}\left(\left\{z_{a}\right\}\right)}\left(M_{A_{a}}\right)\right)-r_{C_{A_{a}}}+2(n-1)\left(C_{A}, X_{\infty}\right)_{\infty_{a}}+1 .
$$

Here $\left(C, C^{\prime}\right)_{\infty_{a}}$ is the intersection number of divisors $C, C^{\prime}$ at $\infty_{a}$.

Proof. We need to compute $\operatorname{dim}_{\mathbb{C}} \operatorname{Hom}_{\mathbb{C}\left(\left(z_{a}\right)\right)}\left(\operatorname{End}_{\mathbb{C}\left(\left(z_{a}\right)\right)}\left(\widetilde{M_{A_{a}}}\right), \mathbb{C}\left(\left(z_{a}\right)\right)\right)^{\text {hor }}$. Let

$$
\operatorname{diag}\left(E_{q_{1}^{a}\left(z_{a}\right), R_{1}^{a}}, \ldots, E_{q_{m_{a}}^{a}\left(z_{a}\right), R_{m_{a}}^{a}}\right)
$$

be the multiplicity free HTL normal form of $M_{A_{a}}$. Then we have the irreducible decomposition

$$
\widetilde{M_{A_{a}}} \cong \bigoplus_{i=1}^{m_{a}} \widetilde{M_{E_{q_{i}^{a}\left(z_{a}\right), R_{i}^{a}}}}
$$


This decomposition shows that

$$
\begin{aligned}
& \operatorname{Hom}_{\mathbb{C}\left(\left(z_{a}\right)\right)}\left(\operatorname{End}_{\mathbb{C}\left(\left(z_{a}\right)\right)}\left(\widetilde{M_{A_{a}}}\right), \mathbb{C}\left(\left(z_{a}\right)\right)\right)^{\text {hor }} \\
& \cong \operatorname{Hom}_{\mathbb{C}\left(\left(z_{a}\right)\right)}\left(\widetilde{M_{A_{a}}}, \widetilde{M_{A_{a}}}\right)^{\text {hor }} \\
& \cong \operatorname{Hom}_{\mathbb{C}\left(\left(z_{a}\right)\right)}\left(\bigoplus_{i=1}^{m_{a}} \widetilde{M_{E_{q_{i}^{a}\left(z_{a}\right), R_{i}^{a}}}} \bigoplus_{i=1}^{m_{a}} \widetilde{M_{E_{q_{i}^{a}\left(z_{a}\right), R_{i}^{a}}}}\right. \text { hor } \\
& \cong \bigoplus_{i=1}^{m_{a}} \bigoplus_{j=1}^{m_{a}} \operatorname{Hom}_{\mathbb{C}\left(\left(z_{a}\right)\right)}\left(\widetilde{M_{E_{q_{i}^{a}\left(z_{a}\right), R_{i}^{a}}}}, \widetilde{M_{E_{q_{j}^{a}\left(z_{a}\right), R_{j}^{a}}}}\right)^{\text {hor }} \\
& \cong \bigoplus_{i=1}^{m_{a}} \operatorname{Hom}_{\mathbb{C}\left(\left(z_{a}\right)\right)}\left(\widetilde{M_{E_{q_{i}^{a}\left(z_{a}\right), R_{i}^{a}}}}, \widetilde{M_{E_{q_{i}^{a}\left(z_{a}\right), R_{i}^{a}}}}\right)^{\text {hor }} \\
& \cong \mathbb{C}^{\oplus m_{a}}
\end{aligned}
$$

by Schur's lemma since $\widetilde{M_{E_{q_{i}^{a}\left(z_{a}\right), R_{i}^{a}}}}$ are irreducible for $i=1,2, \ldots, m_{a}$.

Then the desired equation directly comes from Theorem 3.8 ,

Remark 4.4. Let us introduce the $\delta$-invariant of a singularity which is defined by using the Milnor number as follows,

$$
\delta\left(C_{A_{a}}\right):=\frac{1}{2}\left(\mu\left(C_{A_{a}}\right)+r_{C_{A_{a}}}-1\right) .
$$

See [25] for a geometric meaning of this invariant. Here we note that the germ $C_{A_{a}}$ is reduced by the multiplicity free condition. Then the above formula can be rewritten in a natural form,

$$
2 \delta\left(C_{A_{a}}\right)-2(n-1)\left(C_{A}, X_{\infty}\right)_{\infty_{a}}=-\delta\left(\operatorname{End}_{\mathbb{C}\left(\left\{z_{a}\right\}\right)}\left(M_{A_{a}}\right)\right) .
$$

Theorem 4.5. We use the same notation as above. Suppose that $\nabla_{A}$ satisfies Assumption 4.2 and $C_{A}$ is irreducible. Moreover suppose that $C_{A}$ is smooth on $T^{*} X$. Then the Euler characteristic $\chi\left(\widetilde{C_{A}}\right)$ of the normalization $\widetilde{C_{A}}$ of $C_{A}$ coincides with the index of rigidity of $\nabla_{A}$, i.e.,

$$
\chi\left(\widetilde{C_{A}}\right)=\operatorname{rig}\left(\nabla_{A}\right) .
$$

Proof. Since $C_{A}$ is smooth on $T^{*} X$, possible singularities are only on $C_{A} \cap$ $X_{\infty}=\left\{\infty_{a} \mid a \in D\right\}$. Hence the Euler characteristic $\chi\left(\widetilde{C_{A}}\right)$ can be computed by the formula

$$
\chi\left(\widetilde{C_{A}}\right)=\left(2-2 g_{a}\left(C_{A}\right)\right)+2 \sum_{a \in D} \delta\left(C_{A_{a}}\right)
$$

see Proposition 3 in section IV of [30] for example. We have already computed the arithmetic genus $g_{a}\left(C_{A}\right)$ in the equation (1). Thus

$$
\chi\left(\widetilde{C_{A}}\right)=n^{2}(2-2 g)+(2-2 n)\left(C_{A}, X_{\infty}\right)+2 \sum_{a \in D} \delta\left(C_{A_{a}}\right) .
$$


Finally the formula in Remark 4.4 shows that

$$
\begin{aligned}
\chi\left(\widetilde{C_{A}}\right)= & n^{2}(2-2 g)+(2-2 n)\left(C_{A}, X_{\infty}\right) \\
& +\sum_{a \in D}\left(-\delta\left(\operatorname{End}_{\mathbb{C}\left(\left\{z_{a}\right\}\right)}\left(M_{A_{a}}\right)\right)+2(n-1)\left(C_{A}, X_{\infty}\right)_{\infty_{a}}\right) \\
= & (2-2 g) \operatorname{rank}\left(\mathcal{E} \operatorname{nd}\left(\nabla_{A}\right)\right)-\sum_{a \in D} \delta\left(\operatorname{End}_{\mathbb{C}\left(\left\{z_{a}\right\}\right)}\left(M_{A_{a}}\right)\right)=\operatorname{rig}\left(\nabla_{A}\right) .
\end{aligned}
$$

Corollary 4.6. Let $\nabla_{A}$ be as in Theorem 4.5 and moreover assume that $\nabla_{A}$ is irreducible. Then we have the following numerical coincidences of the cohomology groups,

$$
h_{\mathrm{dR}}^{i}\left(X, j_{! *}\left(\mathcal{E} \operatorname{nd} \nabla_{A}\right)\right)=h^{i}\left(\widetilde{C_{A}}, \mathbb{C}\right), \quad i=0,1,2 .
$$

Here $h^{i}(*):=\operatorname{dim}_{\mathbb{C}} H^{i}(*)$.

Proof. By the irreducibility and duality, we have

$$
h_{\mathrm{dR}}^{0}\left(X, j_{! *}\left(\mathcal{E} \mathrm{nd} \nabla_{A}\right)\right)=h_{\mathrm{dR}}^{2}\left(X, j_{! *}\left(\mathcal{E} \mathrm{nd} \nabla_{A}\right)\right)=1 .
$$

which shows that

$$
h_{\mathrm{dR}}^{i}\left(X, j_{! *}\left(\mathcal{E} \mathrm{nd} \nabla_{A}\right)\right)=1=h^{i}\left(\widetilde{C_{A}}, \mathbb{C}\right)
$$

for $i=0,2$. Thus Theorem 4.5 implies that

$$
h_{\mathrm{dR}}^{1}\left(X, j_{! *}\left(\mathcal{E} \operatorname{nd} \nabla_{A}\right)\right)=2-\operatorname{rig} \nabla_{A}=2-\chi\left(\widetilde{C_{A}}\right)=h^{1}\left(\widetilde{C_{A}}, \mathbb{C}\right) .
$$

Remark 4.7. A similar comparison of Euler characteristics of differential equations and another geometric counterparts, namely $\ell$-adic sheaves, has been known by Katz in [18, [19. Also he pointed out a similarity between local properties, namely, the singularities of differential equations and the ramifications of local Galois actions on $\ell$-adic sheaves. One can find a table of analogies by Katz in [17]. In this table of analogies, the irregularity of a differential equation corresponds to the Swan conductor of the local Galois action on an $\ell$-adic sheaf. On the arithmetic geometry side, the comparison formula of the Swan conductor and the Milnor number has been studied, which is called Deligne's Milnor formula, see [7]. Our formula might give an analogy of this Milnor formula if we follow Katz' table.

\section{REFERENCES}

[1] D. Arinkin, Fourier transform and middle convolution for irregular D-modules. Preprint arXiv:0808.0699. (2008).

[2] D. Babbitt, V. Varadarajan, Formal reduction theory of meromorphic differential equations: a group theoretic view. Pacific J. Math., 109 no. 1, 1-80 (1983).

[3] W. Balser, Formal power series and linear systems of meromorphic ordinary differential equations. Universitext, Springer-Verlag, New York, (2000).

[4] O. Biquard, P. Boalch, Wild non-abelian Hodge theory on curves. Compos. Math., 140 no. 1, 179-204 (2004).

[5] S. Bloch, H. Esnault, Local Fourier transforms and rigidity for $\mathscr{D}$-modules. Asian J. Math., 8 no. 4, 587-605 (2004).

[6] P. Boalch, Symplectic Manifolds and Isomonodromic Deformations, Adv. Math., 163, 137-205 (2001). 
[7] P. Deligne, La formule de Milnor. Groupes de Monodromie en Géométrie Algébrique, SGA 7II, Springer Lecture Note in Mathematics,0 340, 197-211 (1973).

[8] P. Deligne, Equations differentielles á points singuliers reguliers. Lecture Notes in Mathematics, 163 (1970).

[9] R. Dijkgraaf, L. Hollands, P. Sułkowski, Quantum curves and D-modules. preprint arXiv:0810.4157 (2008).

[10] O. Dumitrescu, M. Mulase, Quantization of spectral curves for meromorphic Higgs bundles through topological recursion. Topological recursion and its influence in analysis, geometry, and topology, 179-229, Proc. Sympos. Pure Math., 100, Amer. Math. Soc., Providence, RI, 2018.

[11] R. Hartshorne, Algebraic geometry. Graduate Texts in Mathematics, No. 52. SpringerVerlag, New York-Heidelberg, 1977. xvi+496 pp.

[12] A. Hefez, Irreducible plane curve singularities. Real and complex singularities. 1-120. Lecture Notes in Pure and Appl. Math., 232, Dekker, New York, 2003.

[13] N. Hitchin, The self-duality equations on a Riemann surface. Proc. London Math. Soc. (3) 55 (1987), no. 1, 59-126.

[14] M. Hukuhara, Sur les points singuliers des équation différentielles linéaires. III. Mem. Fac. Sci. Kyusyu Imp. Univ. A. 2 (1942), 125-137.

[15] M. Jimbo, T. Miwa, K. Ueno, Monodromy preserving deformations of linear differential equations with rational coefficients I, Physica 2D, 306-352 (1981).

[16] S. Kamimoto, A relation between structures of turning points and singular points. personal note, (2013).

[17] N. Katz, On the calculation of some differential Galois groups. Invent. Math. 87 (1987), 13-61.

[18] N. Katz, Exponential sums and differential equations. Annals of Mathematics Studies, 124. Princeton University Press, Princeton, NJ, 1990. xii+430 pp.

[19] N. Katz, Exponential sums over finite fields and differential equations over the complex numbers: some interactions. Bull. AMS 23 No.2 (1990), 269-309.

[20] N. Katz Rigid local systems. Annals of Mathematics Studies, vol. 139, Princeton University Press, 1996.

[21] H. Komatsu, On the index of ordinary differential operators. J. Fac. Sci. Univ. Tokyo Sect. IA Math. 18 (1971), 379-398.

[22] M. Kontsevich, Y. Soibelman, Wall-crossing structures in Donaldson-Thomas invariants, integrable systems and Mirror Symmetry. Preprint, arXiv:1303.3253, 2013, 111 pp.

[23] A. Levelt, Jordan decomposition for a class of singular differential operators. Ark. Math. 13 (1975), 1-27.

[24] B. Malgrange, Remarques sur les équations différentielles á points siguliers irréguliers. Lecurete Notes in Mathematics 712, Springer-Verlag 1979, 77-86.

[25] J. Milnor, Singular points of complex hypersurfaces. Annals of Mathematics Studies, No. 61 Princeton University Press, Princeton, N.J.; University of Tokyo Press, Tokyo 1968 iii+122 pp.

[26] T. Mochizuki, Wild harmonic bundles and wild pure twistor D-modules. Astérisque 340, Société Mathématique de France, 2011.

[27] N. Nitsure, Moduli space of semistable pairs on a curve. Proc. London Math. Soc. (3) 62 (1991), no. 2, 275-300.

[28] T. Oshima, Complex linear ordinary differential equations on the Riemann sphere and multivariable hypergeometric functions (in Japanese). Proceedings of 14th Oka Symposium, 2016.

[29] C. Sabbah, An explicit stationary phase formula for the local formal Fourier-Laplace transform. Singularities I, 309-330, Contemp. Math., 474, Amer. Math. Soc., Providence, RI, 2008.

[30] J. P. Serre, Algebraic groups and class fields. Graduate Texts in Mathematics, 117. Springer-Verlag, New York, 1988.

[31] C. Simpson, Higgs bundles and local systems. Inst. Hautes Etudes Sci. Publ. Math. (1992), no. 75, 5-95. 
[32] S. Szabó, The birational geometry of unramified irregular Higgs bundles on curves. Internat. J. Math. 28 (2017), no. 6, 1750045, 32 pp.

[33] H. Turrittin, Convergent solutions of linear homogeneous differential equations in the neighborhood of an irregular singular point. Acta. Math. 93, (1955), 27-66.

[34] C. Wall, Singular points of plane curves. London Mathematical Society Student Texts, 63. Cambridge University Press, Cambridge, 2004. xii+370 pp.

[35] E. Witten, Gauge theory and wild ramification. preprint arXiv:0710.0631 (2007).

Email address: kazuki@math.s.chiba-u.ac.jp

Department of Mathematics and Informatics, Chiba University, 1-33, YayoiCho, InAGe-KU, ChibA-Shi, ChibA, 263-8522 JAPAN 